\title{
Cathode Lens Mode of the SEM in Materials Science Applications
}

\author{
Luděk Frank $^{1}$, Ilona Müllerová ${ }^{1}, K_{\text {Kenji Matsuda }}^{2}$ and Susumu Ikeno ${ }^{2}$ \\ ${ }^{1}$ Institute of Scientific Instruments, Academy of Sciences of the Czech Republic, CZ-61264 Brno, Czech Republic \\ ${ }^{2}$ Dept. Materials Science and Engineering, Faculty of Engineering, University of Toyama, Toyama 930-8555, Japan
}

The cathode lens (CL) mode of the SEM, employing sample as a cathode of the beam-decelerating electrostatic lens, enables one to preserve the image resolution down to lowest electron energies and in the same time secures an excellent collection efficiency of signal species. In the range of tens and units of $\mathrm{eV}$, new image contrasts become available, based on the quantum mechanical character of scattering and the electron wavelength comparable with inter-atomic distances. However, already in the low keV and hundreds of eV ranges the CL mode has proven itself very efficient in many materials science applications, overcoming some weak points the conventional SEM modes suffer from. Selected material structures are presented as demonstration examples. [doi:10.2320/matertrans.48.944]

(Received November 16, 2006; Accepted February 13, 2007; Published April 25, 2007)

Keywords: electron microscopy of materials, scanning electron microscopy, low energy electron microscopy, cathode lens

\section{Introduction}

The scanning electron microscope (SEM) represents a well-established tool for observation and microanalysis of solid surfaces and thin surface films. When scanning the field of view with a focused electron probe and collecting some part of the emitted signals, we automatically ascribe the acquired information to immediate coordinates of the primary electron (PE) beam. However, the full interaction volume, from which signal species are released, is significantly larger than the primary spot size in the surface plane of the sample, usually considered defining the image resolution. Dimensions of the information producing volume depend on the beam energy and at standard SEM energies they reach units of micrometers or even more. Still, images at a resolution similar to the spot size can be recorded but only when the utmost surface bound contribution to the image signal dominates enough. An example is the surface topography visualized via slow secondary electrons excited by PE (so called SE1) within a tiny, directly illuminated spot but combined with the SE2 signal released by backscattered electrons (BSE) upon their return toward the surface. Because of lateral diffusion, BSE "illuminate" a significantly larger area, releasing hence SE2 from a spot smeared so much that this contrast component may "dissolve" in the image background.

At many instances the surface relief does not dominate like when examining smooth flat surfaces with small localized inhomogeneities (inclusions, precipitates, etc.). In this case the image resolution deteriorates drastically. Another issue in the SEM practice is that standard signal detection principles produce single-channel data only and, moreover, definition of the measured values, i.e. demarcation of the energy and angular intervals acquired from full distributions of the electron emission, is insufficient or even unknown.

In order to overcome at least some of these issues, it suggests itself to lower the energy of incident electrons, diminishing hence the interaction volume. Practicians' experience has shown energies below, say, $5 \mathrm{keV}$ advantageous as regards appearance of surface topography details, local charging of less conductive samples, and yield of secondary electrons. Further motivations to lowering the beam energy include reduced overbrightening of steeply inclined surface facets (so called edge effect), which is important when e.g. semiconductor structures are examined.

Historical milestones on the way to the low energy SEM were at its beginning connected with developments in the emission electron microscopy (EEM). In an EEM, slow signal electrons are accelerated in a strong homogeneous electric field immediately above the emitting surface. In this configuration, lower the electron energy in the sample plane, better the final image resolution, ${ }^{1)}$ which is a relation just opposite to what holds in the conventional SEM. An important progress step was introduction of the low energy electron microscope (LEEM) as an EEM with the emission excited by a coherent planar wave of incident slow electrons. ${ }^{2,3)}$

The on-specimen electric field was, nevertheless, proposed to be implemented even in one of the first SEMs but no practical results were presented that time. ${ }^{4)}$ First micrographs, albeit of a low resolution, appeared much later but still long time ago. ${ }^{5)}$ Intensive development of the method started then with the 1990's.

The cathode lens (CL) mode of the SEM (the SLEEMscanning low energy electron microscope) $)^{6}$ enables one to decrease the landing energy of electrons while preserving the spot size, and modifies the conditions on which the signal electrons are collected so its materials science applications are worth of reviewing.

\section{Scattering of Slow Electrons in Solids}

Specific features of microscopy with slow electrons in any version follow from electron scattering phenomena in solids, as they are modified when lowering the impact energy. Quantities suitable for a first glance assessment are the crosssections of elastic $\left(\sigma_{e l}\right)$ and inelastic $\left(\sigma_{i n}\right)$ scattering. At high energies, $\sigma_{e l}$ is proportional to atomic number of the target material, providing a source of the broadly utilized material contrast in SEM images. This monotonous dependence is, nevertheless, lost at low energies when quantum mechanical scattering phenomena start to intensify and scattering on the 

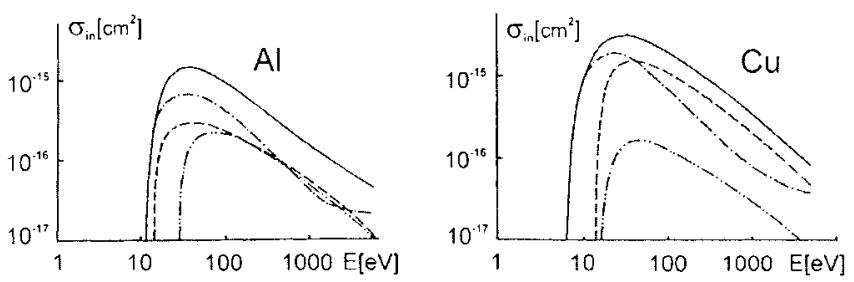

Fig. 1 Calculated total inelastic cross-sections (-) and their main contributions, namely the electron-electron scattering (---.), shell ionization (- - ) and plasmon excitation $(-\cdot \cdot-\cdot \cdot))^{7)}$

complete (anizotropic) crystal potential substitutes for that on individual ion cores. This conversion demonstrates itself in the energy dependence of $\sigma_{e l}$, which becomes "undulating" in a way specific to each particular crystal but generally keeps growing down to the total reflection at zero impact energy.

Contrary to this, the inelastic cross-sections for individual scattering mechanisms culminate somewhere in the range of tens of $\mathrm{eV}$ and then steeply fall (Fig. 1). Such behavior suggests an energy threshold like $50 \mathrm{eV}$ as a boundary between the low and very low energy ranges. Both these ranges are routinely available in the LEEM, with the very low energy (VLE) range utilized dominantly, but in the SEM even the low energy (LE) range has not been fully covered as yet.

The other practically important quantities like, for example, the electron range can be derived from the cross-sections. The electron range at units of $\mathrm{eV}$, in particular its expected steep extension, remains, however, questionable. Evidences have been presented for the increase ${ }^{3)}$ but some other experiments have not provided the same result. ${ }^{8)}$ Obviously, the feature is extremely specimen specific and depends first of all on crystallinity of the sample throughout its full thickness including the very surface.

The carbon contamination traces of electron beam impact as the most often met radiation damage phenomenon gain in intensity when passing the LE range. While every incident electron delivers less energy and even the energy export via the SE emission grows (with stagnating emission of here more important energetic BSEs), the penetrated layer becomes thinner so the spatial density of dissipated energy increases. This adverse tendency stops only around the LE/ VLE threshold, below which all radiation damage effects fade down.

\section{Image Signals Excited by Slow Electrons}

Traditional distinction between SE and BSE emissions, putting a negotiated energy threshold at $50 \mathrm{eV}$, naturally loses it sense when approaching this energy. However, already in the LE range changes are noticeable with respect to what is known from the standard SEM practice for each of these basic signals. Moreover, in the cathode lens field the complete energy spectrum of electron emission is accelerated and hence shifted along the energy scale. Finally, we get the signal beam of an energy spread being equal to the landing energy of the primary beam, with upper limit of the spread interval coinciding with the original primary energy.

Toward low energies the SE yield monotonously grows for

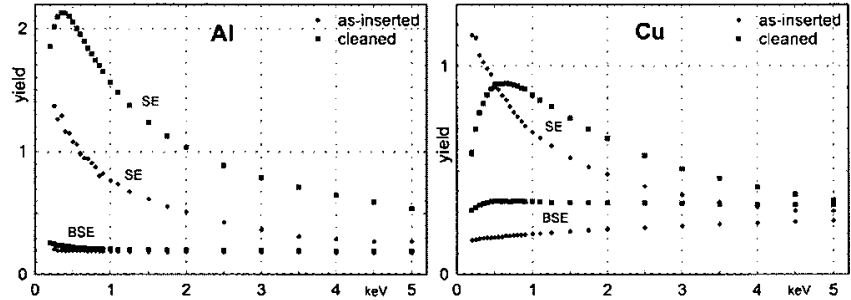

Fig. 2 SE and BSE yields for $\mathrm{Al}$ and $\mathrm{Cu}$ measured under UHV conditions in both as-inserted and in-situ ion beam cleaned status. ${ }^{10)}$

all materials up to a maximum appearing usually within the range of hundreds of eV (for insulators somewhat higher) and mostly exceeding the unit level. When adding the BSE emission, we get the total yield nearly always above one emitted electron per one incident electron. Thus, in the LE range the total yield curve two times crosses the unit level at so-called critical energies. At critical energies, from which the higher is self-adjusting, no charge is dissipated in the sample and non-charging microscopy of insulators is possible. ${ }^{9)}$ Naturally, with the growing SE emission the signal-tonoise ratio in micrographs generally improves.

Further important factor is the penetration of primary electrons to below the escape depth of secondary electrons, which takes place at high energies and, being the cause for steep signal increases at tilted facets, adversely exhausts the dynamical range of image signal. At a certain primary energy, dependent on the target material and situated near to energy at which the SE emission reaches its maximum, penetration and escape depths equilibrate, eliminating hence the edge effect. In the VLE range the SE emission gradually drops but keeps being capable of visualizing the surface topography via tiny ridges and protrusions, which may be just "filled" with the interaction volume of PE.

Along the LE range the BSE yield losses its proportionality to the atomic number of the target so usual material contrast is no more reliable. Still, the BSE emission depends on the target material but influence of its electronic structure combines with that of ion cores and also 3D anisotropy of scattering enhances according to anisotropy of the overall crystal potential as mentioned in the previous paragraph. Consequently, already in the LE range the BSE emission provides combined compositional/structural information not always easy to encrypt without complementary data being available. An apparent effect is the growing contrast of grains in polycrystals. Also the monotonous increase in BSE emission with the impact angle of PE is replaced with more complicated dependences specific to a target structure. Otherwise, even between target components differing only slightly in atomic numbers high BSE contrast can be acquired at a tailored PE energy. Shallow penetration depth of PE means enhanced surface sensitivity also as regards the BSE emission, which in the standard SEM is considered a bulk signal (see Fig. 2). Thus, any surface layers, coatings and contaminations become visible not only in SE micrographs but also in the BSE ones.

Very important for materials science applications is the interaction volume of $\mathrm{PE}$ and its diminishing itself. This otherwise trivial effect is worth of being illustrated (Fig. 3) 


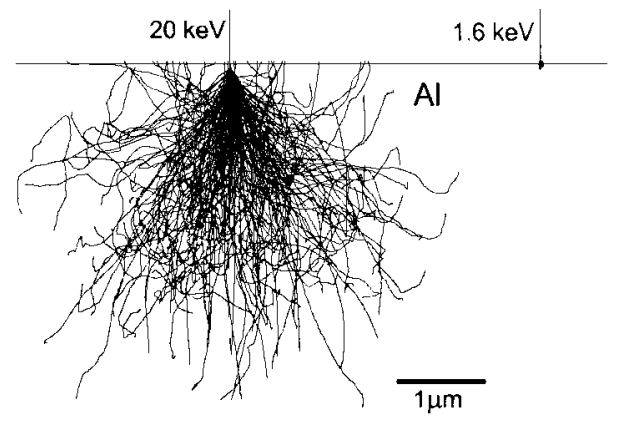

Fig. 3 Monte-Carlo simulated scattering charts in Al for two energies of electron impact (simulation software of D.C. Joy ${ }^{11)}$ was used).

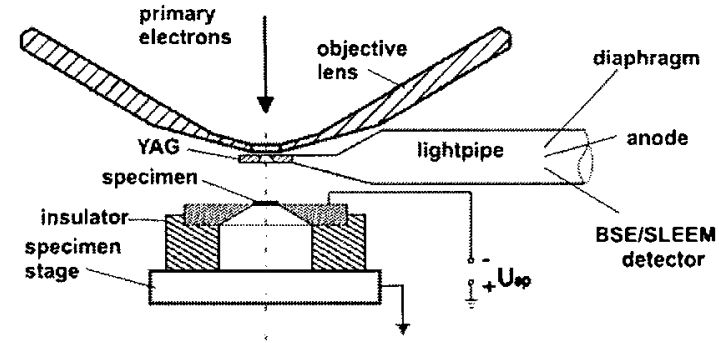

Fig. 5 Scheme of the SLEEM detector placed coaxially below the objective lens.
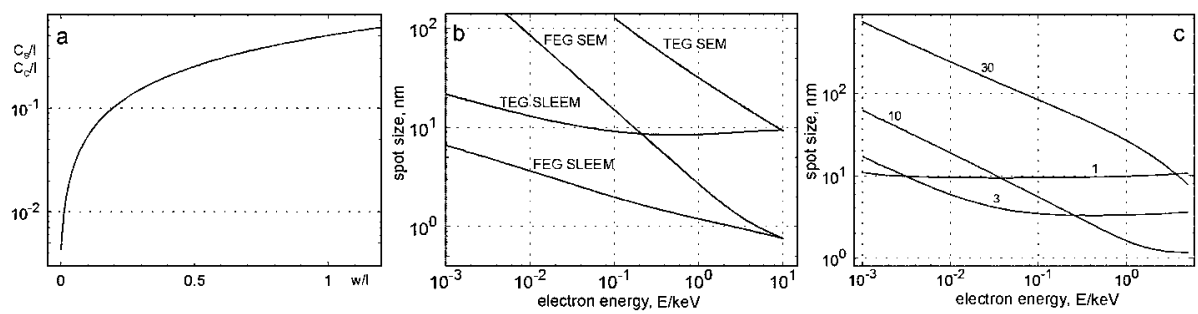

Fig. 4 (a) Approximated values of the aberration coefficients, $C_{S} \approx C_{C}$, in dependence on the working distance $w$ of an immersion lens modeled with two thin electrodes at a distance of $l$ and abrupt field transitions; ${ }^{12}$ (b) energy dependence of the spot size calculated for two typical classes of the SEM, i.e. a traditional thermo-emission gun equipped SEM and that with a cold field emission gun (optimum angular aperture is considered at each impact energy); (c) energy dependence of the spot size calculated at four fixed angular apertures (taken in between the objective and cathode lenses and labeled in mrad) for a typical SEM with the Schottky emission gun.

by simulation of electron trajectories inside the target, ${ }^{11)}$ clearly revealing a sub-micrometer size inhomogeneity located just in the surface plane as not sharply viewable at standard SEM energies but well reproduced in the LE range.

\section{The SLEEM Mode}

Principle of the cathode lens or SLEEM mode of the SEM is quite simple: the specimen is held on a high negative potential with respect to the ground, comparable with the potential applied to the gun cathode. Then, the primary beam is impinging on the sample with energy given by difference of both these biases and can be arbitrarily adjusted by varying the sample bias. Demands on the voltage supply for biasing the sample include then stability similar to that of the main acceleration voltage supply, and also very fine adjustability. Alternative solution is to bias the sample with respect to the gun cathode, reducing so drifts and fluctuations in the beam energy not combined from two supplies any more. Advantageous is, however, to have possibility of a negative impact energy (the mirror mode) requiring the single voltage supply passing smoothly zero, which is rather difficult task. More details about the SLEEM mode can be found in comprehensive review. ${ }^{6)}$

As already mentioned in the introduction, the CL aberrations improve when decreasing the electron energy on the low energy side of the CL. In the SEM application, it means a decrease in aberration coefficients with decreasing electron energy. Figure 4(a) shows that the zero working distance of a beam retarding immersion electrostatic lens (i.e. the cathode lens with the sample itself serving as the cathode) means significant reduction in aberration coefficients. This aberra- tion correcting ability of CL is one of crucial prerequisites of the SLEEM performance, the consequence of which is very favorable energy dependence of the spot size (Fig. 4(b)). Even when abandoning the possibility of adjusting the optimum angular aperture for each impact energy and using some fixed aperture in front of CL, we get both the LE and VLE spot sizes much smaller than in the standard SEM mode of a given instrument (Fig. 4(c)).

Second key factor is collimation of the signal species to a relatively narrow "signal beam", which is normally centered to the optical axis but can be also moderately inclined when slightly tilting the sample. The homogeneous part of the CL field increases the initial axial velocity of electrons so that they follow a parabolic trajectory and in the anode plane they fill a circle of a radius $2 l /(k-1)^{1 / 2}$, where $l$ is the cathode/ anode distance and $k$ is the immersion ratio (ratio of final to initial energies). Thus, the complete emission is collimated to a spot few mm in size or less, which can be easily collected or passed through the objective lens bore to a detector situated there. Moreover, in this signal beam we get both secondary and backscattered electrons including slow BSE and also BSE emitted at high angles to the optical axis, which are not acquired by standard BSE detectors. This ability of the cathode lens creates extremely high collection efficiency near to $100 \%$ with acquisition of normally not available signal components, and represents the second keystone of the SLEEM performance.

One of possible implementations of the SLEEM mode employs a coaxial bored scintillator disc as the core component of an assembly inserted to below the objective lens (OL) (Fig. 5). ${ }^{6,12)}$ This detector type is similar to the Autrata-type BSE detector ${ }^{13)}$ and with the unbiased sample it 
also detects the conventional BSE signal. However, here the scintillator bore should be very small in order to reduce the part of slowest electrons escaping detection through the bore-good compromise is a diameter around $300 \mu \mathrm{m}$ (see discussion in the review ${ }^{6)}$ ). Small opening and large surrounding electric field requires the detector to be routinely finely adjustable in all three axes. The lateral adjustment is significantly facilitated when also the upper surface of the scintillator is left active so that the central bore is directly observed at low magnifications. Also homogeneity of the CL field should be adjustable by two independent, mutually perpendicular tilts either both applied to the sample or possibly with one of them performed with the detector in the form of its rotation around the main axis.

The below-OL detection is relatively easy adapted to commercial SEMs ${ }^{14)}$ and hence represents a solution available for the time before the SLEEM mode appears on the instrument market. However, its main drawbacks, i.e. loss of the central part of the signal beam including specular diffraction spot at lowest energies and consumption of few $\mathrm{mm}$ of the working distance, suggest a through-the-lens detector type with deflection of the signal beam off the optical axis the optimum solution. With any of these implementations one has to take into account the optical power of the cathode lens and correct for this procedures determining in the SEM control software the magnification and focusing of the OL. For the CL inserted to below OL, i.e. for sequentially arranged electric and magnetic fields, the correcting algorithms can be expressed analytically to a good accuracy. ${ }^{15}$ ) The maximum correction to magnification is around $40 \%$ while that to the object distance of the OL does not exceed $30 \%$.

\section{Selected Application Results}

Because of low availability of adapted instruments the volume of experience collected so far is limited to several demonstration experiments having been performed in order to map the SLEEM mode performance on larger families of samples. Specific features of the LE and VLE electron scattering together with properties of the detector type usually used (see Fig. 5) result in significant modifications of the image contrasts known and expected with such typical samples. Thus, it is recommendable to compare always the SLEEM mode micrographs with images acquired in standard SEM modes on the same field of view. The micrographs below were acquired in various TEG SEM instruments adapted to the SLEEM mode in the same way.

In Fig. 6 we can assess contrast modifications caused by diminished interaction volume, combination of the SE and BSE signals and scattering anisotropy combined with the given detector acceptance, as they appear on small precipitates leveled with the surface plane. In this case the SLEEM study enabled us to distinguish between two types of precipitates from which one exhibit a dark core with intensive bright frame around. More detailed study ${ }^{16,17)}$ showed for this type the $\mathrm{Mg} / \mathrm{Si}$ ratio different from $2: 1$ met with the $\beta$-phase, namely changed near to $3: 1$. Very strong SLEEM contrast between frame and core indicates existence of some transition layer between matrix and the
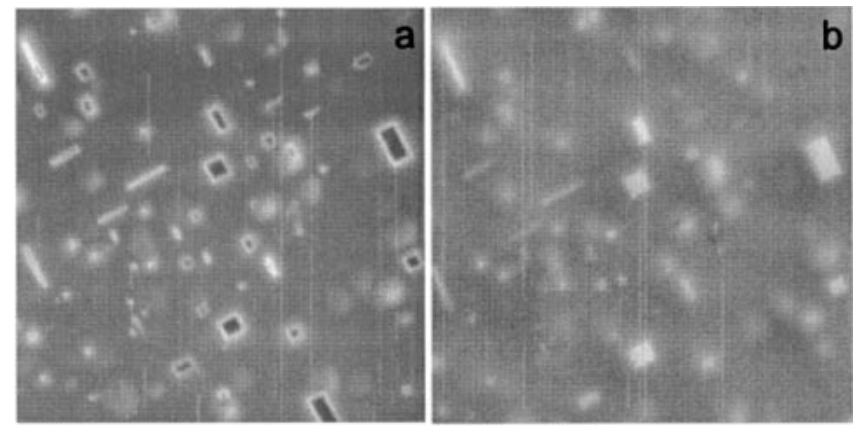

Fig. $6 \mathrm{Mg}_{\mathrm{x}} \mathrm{Si}$ precipitates in the Al-1.0 mass $\% \mathrm{Mg}_{2} \mathrm{Si}-0.4$ mass\% $\mathrm{Mg}$ alloy: (a) SLEEM mode at the landing energy of $1600 \mathrm{eV}$ and primary energy $10 \mathrm{keV}$, (b) standard SE image at $10 \mathrm{keV}$; width of the field of view is $7 \mu \mathrm{m}$.

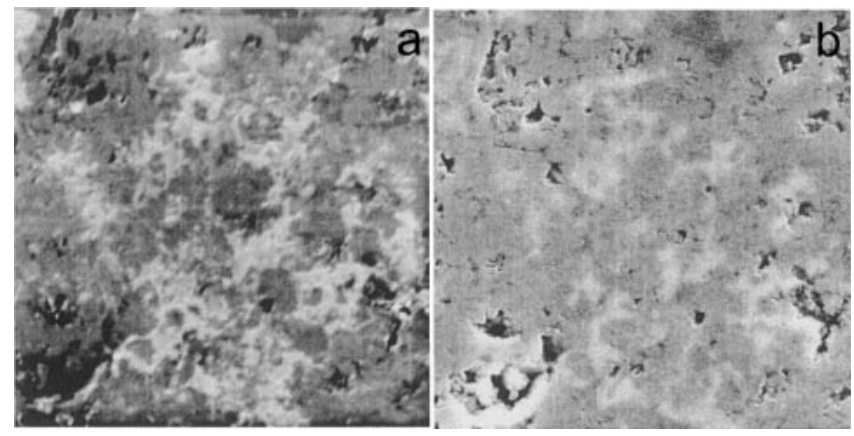

Fig. $7 \mathrm{MgB}_{2} / \mathrm{Al}$ composite: (a) SLEEM mode at the landing energy of $10 \mathrm{eV}$, (b) standard SE image at $10 \mathrm{keV}$; width of the field of view is $14 \mu \mathrm{m}$.

precipitate core. This layer differs in its composition and structure so that its emission, most probably the BSE one, is strongly enhanced. Additional experiments, best with high resolution TEM, are needed to analyze this layer. In Fig. 7 the structure is composed of ceramic particles spread in a light metal matrix composite. The particles are much sharper at low energies, of course, but also their contrast is enhanced. The reason is in difference in the elastic BSE yields, which increases down to very low energies. As distinct to the previous cases, Figure 8 presents a structure with small geometrical shapes protruding from the average surface, which are also not well observable at higher energies. Here again the diminished interaction volume secures sharp edges in images of the crystals while absence of lowest polar angle emitted electrons in the detected signal leads to planar contrasts between mutually tilted facets. Generally, alumina particles with the spinel crystals, i.e. the oxides, are brighter than the matrix at $10 \mathrm{keV}$ where the SE contribution to the image signal dominates while at $1 \mathrm{keV}$ the contrast inverts because of lower BSE signal from them.

In Fig. 9 the "non-charging" mode at the second (higher) critical energy is demonstrated on a non-conductive structure, which is normally heavily charged when observed at conventional SEM energies. Furthermore, the material is inhomogeneous because of carbon particles spread in the polymer matrix, which might complicate using the critical energy method. However, both components have similar critical energies and typical heterogeneity dimensions are 


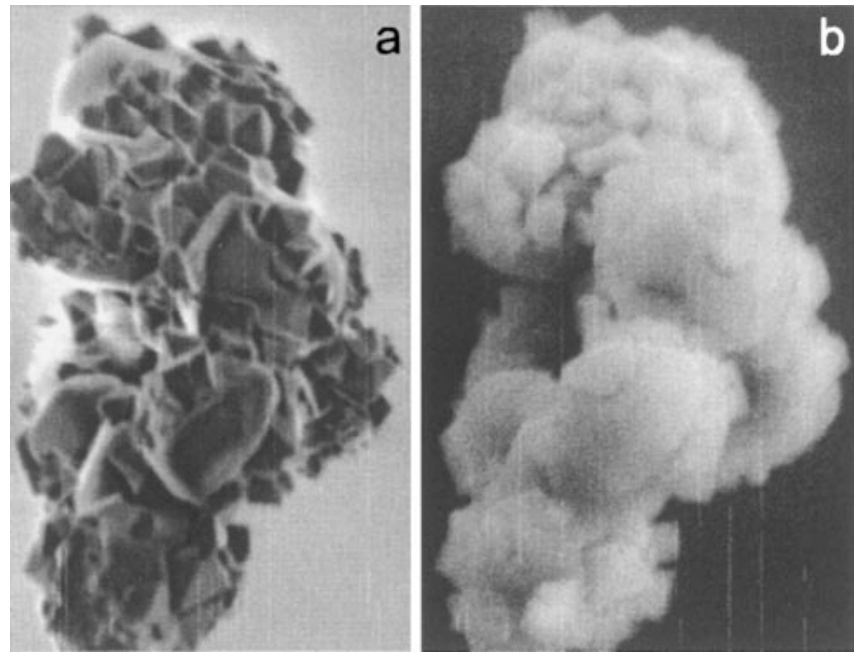

Fig. $8 \mathrm{MgAl}_{2} \mathrm{O}_{4}$ crystals grown on the surface of an $\mathrm{Al}_{2} \mathrm{O}_{3}$ particle in the 4 vol\% $\mathrm{Al}_{2} \mathrm{O}_{3} / \mathrm{Al}-1.0$ mass $\% \mathrm{Mg}_{2} \mathrm{Si}$ metal-matrix composite: (a) SLEEM mode at the landing energy of $1 \mathrm{keV}$ and primary energy $10 \mathrm{keV}$, (b) standard SE image at $10 \mathrm{keV}$; width of the field of view $3 \mu \mathrm{m}$.

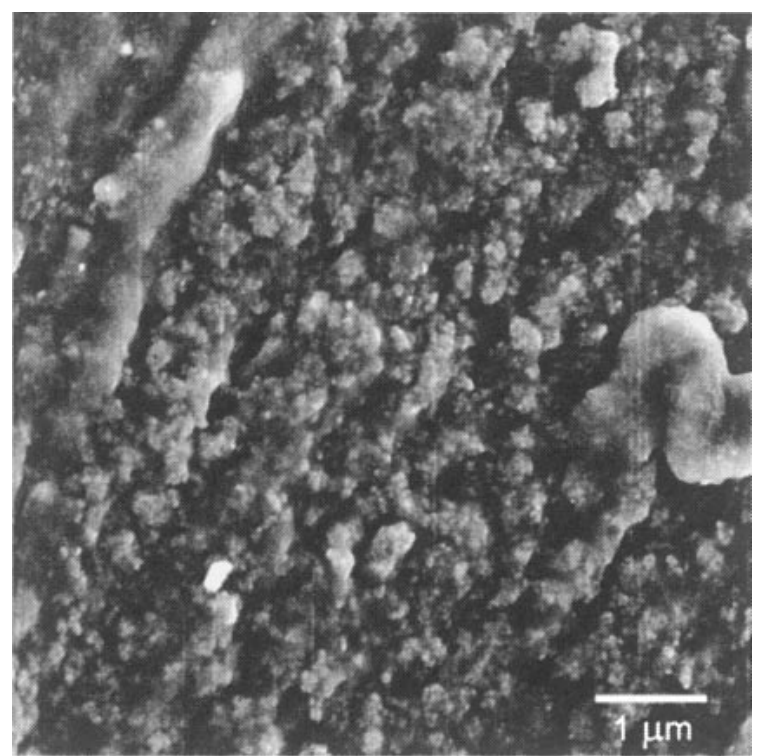

Fig. 9 Cross-section of the black rubber: SLEEM in the non-charging adjustment (the "critical" energy), landing electron energy $4.3 \mathrm{keV}$, primary energy $10 \mathrm{keV}$.

quite small. In this case only very low local surface potentials are generated and we are able to establish one energy value for the entire field of view.

Let us underline that within the energy range accessible for other low voltage SEM types, i.e. standard columns with the electron energy constant between the gun anode and the sample or configurations using the "booster" (e.g. so-called Gemini lens) the main dissimilarity of the SLEEM mode consists in collection efficiency of the below-OL detector combined with the cathode lens. This detector type is capable of acquiring also low energy BSE and BSE emitted at large angles with respect to the surface normal, which are normally not available for the SEM imaging. It is even not easy, although not impossible, to detect those BSE by through-thelens detectors. This is main reason for success of the SLEEM mode in the configuration described here as regards ability of acquiring new contrast types.

\section{Conclusions}

The cathode lens mode (the SLEEM mode) enables one to decrease arbitrarily the landing energy of electrons in a SEM while preserving the image resolution similar to that achievable at tens of keV. Very slow electrons at tens and units of $\mathrm{eV}$ with their high surface sensitivity and quantum mechanical nature of scattering mechanisms are extremely useful in examination of very clean and well defined crystallinic surfaces. However, the cathode lens assembly with its impact on both primary and signal electrons affects markedly also image contrasts available in the ranges of hundreds $\mathrm{eV}$ and low $\mathrm{keV}$ and provides new types of information. This paper offers description of the SLEEM mode and several examples of its use.

\section{Acknowledgements}

This work is supported by the Czech Science Foundation under grants no. 102/05/2327 (I.M.) and 202/04/0281 (L.F.). The study of aluminum based alloys is made in the frame of the 2006 Research Project supported by the Venture Business Laboratory of the University of Toyama.

\section{REFERENCES}

1) A. Recknagel: Zeitschr. Phys. 117 (1941) 689-708.

2) E. Bauer: Proc. 5th Int. Congr. Electron Microsc., vol. 1, ed. by S. S. Bresse, jr., (Academic Press, New York, 1962) pp. D11-12.

3) E. Bauer: Repts Progr. Phys. 57 (1994) 895-938.

4) V. A. Zworykin, J. Hillier and R. L. Snyder: ASTM Bull. 117 (1942) 15-23.

5) R. S. Paden and W. C. Nixon: J. Phys. E: Sci. Instrum. 1 (1968) 10731080.

6) I. Müllerová and L. Frank: Adv. Imaging and Electron Phys. 128 (2003) 309-443.

7) Y. C. Ho, Z. Y. Tan, X. L. Wang and J. G. Chen: Scanning Microsc. 4 (1991) 945-951.

8) P. Hrnčiřík and I. Müllerová: Imaging and Microscopy 6 (2004) 47-49.

9) L. Frank, M. Zadražil and I. Müllerová: Scanning 23 (2001) 36-50.

10) M. Zadražil, M. M. El-Gomati and A. Walker: J. Comput. Assist. Microsc. 9 (1997) 123-124.

11) D. C. Joy: Monte Carlo Modeling for Electron Microscopy and Microanalysis, (Oxford University Press, London, 1995).

12) L. Frank and I. Müllerová: J. Electron Microsc. 48 (1999) 205-219.

13) R. Autrata: Scanning Microsc. 3 (1989) 739-763.

14) I. Müllerová and L. Frank: Scanning 15 (1993) 193-201.

15) J. Zobačová, M. Zobač, M. Oral, I. Müllerová and L. Frank: Scanning 28 (2006) 155-163.

16) K. Matsuda, Y. Ishida, I. Müllerová, L. Frank and S. Ikeno: J. Mater. Sci. 41 (2006) 2605-2610.

17) K. Matsuda, S. Ikeno, I. Müllerová and L. Frank: J. Electron Microsc. 54 (2005) 109-117. 